PROCEEDINGS OF THE

AMERICAN MATHEMATICAL SOCIETY

Volume 140, Number 2, February 2012, Pages 541-551

S 0002-9939(2011)11401-1

Article electronically published on September 29,2011

\title{
RADIAL SYMMETRY AND DECAY RATES OF POSITIVE SOLUTIONS OF A WOLFF TYPE INTEGRAL SYSTEM
}

\author{
YUTIAN LEI AND CHAO MA
}

(Communicated by Matthew J. Gursky)

ABSTRACT. In this paper, we study the properties of the positive solutions of a nonlinear integral system involving Wolff potentials:

$$
\left\{\begin{array}{c}
u_{1}=W_{\beta, \gamma}\left(f_{1}(u)\right) \\
\vdots \\
u_{m}=W_{\beta, \gamma}\left(f_{m}(u)\right),
\end{array}\right.
$$

where $u=\left(u_{1}, \ldots, u_{m}\right)$ and

$$
W_{\beta, \gamma}(f)(x)=\int_{0}^{\infty}\left[\frac{\int_{B_{t}(x)} f(y) d y}{t^{n-\beta \gamma}}\right]^{\frac{1}{\gamma-1}} \frac{d t}{t}
$$

with $1<\gamma<2$ and $n>\beta \gamma$. First, we estimate the decay rate of the positive solutions at infinity. Based on this, we prove radial symmetry and monotonicity for those solutions by the refined method of moving planes in integral forms, which was established by Chen, $\mathrm{Li}$ and Ou. Since the Kelvin transform cannot be used in such a Wolff type system, we have to find a new technique to study the asymptotic estimate, which is essential when we move the planes.

\section{INTRODUCTION}

In this paper, we will study the abstract integral system involving the Wolff potentials

$$
\left\{\begin{array}{c}
u_{1}=W_{\beta, \gamma}\left(f_{1}(u)\right) \\
\vdots \\
u_{m}=W_{\beta, \gamma}\left(f_{m}(u)\right),
\end{array}\right.
$$

where $u_{i}>0(i=1, \ldots, m), u=\left(u_{1}, \ldots, u_{m}\right)$, and $f_{i}(u(x))=C \prod_{i=1}^{m} u_{i}^{e_{i}}(x)$ with $e_{1}+\cdots+e_{m}=p:=\frac{(n+\gamma \beta)(\gamma-1)}{n-\gamma \beta}$. In the following, we will always assume $p>1$ and $e_{i} \geq 0$.

Received by the editors November 3, 2010.

2010 Mathematics Subject Classification. Primary 35J50, 45E10, 45G05.

Key words and phrases. Integral equation, Wolff potential, decay rate, radial symmetry.

(C)2011 American Mathematical Society Reverts to public domain 28 years from publication 
The Wolff potential of a nonnegative function $f \in L_{l o c}^{1}\left(R^{n}\right)$ is defined as (cf. [13])

$$
W_{\beta, \gamma}(f)(x)=\int_{0}^{\infty}\left[\frac{\int_{B_{t}(x)} f(y) d y}{t^{n-\beta \gamma}}\right]^{\frac{1}{\gamma-1}} \frac{d t}{t},
$$

where $1<\gamma<\infty, \beta>0, \beta \gamma<n$, and $B_{t}(x)$ is the ball of radius $t$ centered at $x$. It is not difficult to see that $W_{1,2}(f)$ is Newton's potential and $W_{\frac{\alpha}{2}, 2}(f)$ is Reisz's potential.

Recalling the work in [17, [18] and [28, we know that the Wolff potential is helpful for studying the nonlinear PDEs. For example, $W_{1, p}(\omega)$ and $W_{\frac{2 k}{k+1}, k+1}(\omega)$ can be used to estimate the solutions of the $p$-Laplace equation

$$
-\operatorname{div}\left(|\nabla u|^{p-2} \nabla u\right)=\omega
$$

and the $k$-Hessian equation

$$
F_{k}[-u]=\omega, \quad k=1,2, \ldots, n,
$$

respectively. Here $F_{k}[u]=S_{k}\left(\lambda\left(D^{2} u\right)\right), \lambda\left(D^{2} u\right)=\left(\lambda_{1}, \lambda_{2}, \ldots, \lambda_{n}\right)$ with $\lambda_{i}$ being eigenvalues of the Hessian matrix $\left(D^{2} u\right)$, and $S_{k}(\cdot)$ is the $k$-th symmetric function:

$$
S_{k}(\lambda)=\sum_{1 \leq i_{1}<\cdots<i_{k} \leq n} \lambda_{i_{1}} \lambda_{i_{2}} \cdots \lambda_{i_{k}} .
$$

Two special cases are $F_{1}[u]=\Delta u$ and $F_{n}[u]=\operatorname{det}\left(D^{2} u\right)$.

When $\gamma=2$ and $\beta=\alpha / 2$, (11) becomes

$$
\left\{\begin{aligned}
u_{i}(x) & =\int_{R^{n}} \frac{\left(f_{i}(u(y))\right) d y}{|x-y|^{n-\alpha}}, \quad x \in R^{n}, \quad m=1,2, \ldots, m, \\
u & =\left(u_{1}, u_{2}, \ldots, u_{m}\right), \quad 0<\alpha<n .
\end{aligned}\right.
$$

Here $f_{i}(u), 1 \leq i \leq m$, are real-valued functions of homogeneous degree $\frac{n+\alpha}{n-\alpha}$ and are monotone nondecreasing with respect to all the independent variables $u_{1}, u_{2}, \ldots, u_{m}$. Chen and Li proved the radial symmetry of positive solutions (cf. [6]), and the decay rates were obtained in Section 3 of [6] by the Kelvin transform.

When $m=2, f_{1}(u, v)=u^{p}$ and $f_{2}(u, v)=v^{q}$, (1) becomes

$$
\left\{\begin{array}{l}
u(x)=W_{\beta, \gamma}\left(v^{q}\right)(x), \\
v(x)=W_{\beta, \gamma}\left(u^{p}\right)(x),
\end{array}\right.
$$

which was studied by Ma, Chen and Li (cf. [5] and [26]). Here $p$ and $q$ satisfy

$$
\frac{\gamma-1}{p+\gamma-1}+\frac{\gamma-1}{q+\gamma-1}=\frac{n-\beta \gamma}{n} \text {. }
$$

It turns out that the positive solutions of (3) are radially symmetric and decreasing about some point in $R^{n}$.

Letting $\gamma=2$ and $\beta=\alpha / 2$ in (3), we obtain a Hardy-Littlewood-Sobolev (HLS) type integral system

$$
\left\{\begin{array}{l}
u(x)=\int_{R^{n}} \frac{v^{q}(y) d y}{|x-y|^{n-\alpha}}, \\
v(x)=\int_{R^{n}} \frac{u^{p}(y) d y}{|x-y|^{n-\alpha}} .
\end{array}\right.
$$


Chen, $\mathrm{Li}$ and $\mathrm{Ou}$ used an integral form of the method of moving planes to prove the radial symmetry of the positive solutions of (4) (cf. [7]). Moreover, if $p=q$, then $u=v$, and hence (4) reduces to a single equation (cf. [8]):

$$
u(x)=\int_{R^{n}} \frac{u^{\frac{n+\alpha}{n-\alpha}}(y) d y}{|x-y|^{n-\alpha}} .
$$

The radial symmetry of the positive solutions was obtained in [8] and 24].

For a general weighted HLS type integral system (WHLS)

$$
\left\{\begin{array}{l}
u(x)=\frac{1}{|x|^{\alpha}} \int_{R^{n}} \frac{v^{q}(y) d y}{|y|^{\beta}|x-y|^{n-\alpha}}, \\
v(x)=\frac{1}{|x|^{\beta}} \int_{R^{n}} \frac{u^{p}(y) d y}{|y|^{\alpha}|x-y|^{n-\alpha}},
\end{array}\right.
$$

the radial symmetry of the positive solutions is proved by Jin and Li (cf. 44). Afterwards, they also derived the integrability intervals of those solutions in [14]. Based on this result, Li, Lim and Lei, Ma obtained the asymptotic estimates when $|x| \rightarrow 0$ and $|x| \rightarrow \infty$ (cf. [22], 20]).

In this paper, we will also prove the radial symmetry and estimate the decay rates of positive solutions $u_{i}$ of (1) if $u_{i} \in L^{p+\gamma-1}\left(R^{n}\right)$. We state the main result of this paper, which will be proved in Section 3.

Theorem 1.1. Assuming $u_{i} \in L^{p+\gamma-1}\left(R^{n}\right)$ solves (1), $i=1,2, \ldots, m$, and assuming $f_{i}$ is simple and monotone nondecreasing with respect to the variables $u_{1}, u_{2}, \ldots, u_{m}, f$ is simple means that:

$$
\left(f_{i_{1}}(u), \ldots, f_{i_{k}}(u)\right) \neq\left(f_{i_{1}}(v), \ldots, f_{i_{k}}(v)\right)
$$

whenever

$$
\left(u_{i_{1}}, \ldots, u_{i_{k}}\right)=\left(v_{i_{1}}, \ldots, v_{i_{k}}\right)
$$

and

$$
u_{i_{k+1}}>v_{i_{k+1}}, \ldots, u_{i_{m}}>v_{i_{m}} .
$$

Then $u_{i}(x)$ is radially symmetric and decreasing about some point $x_{0} \in R^{n}$.

In order to use the moving plane method to prove the radial symmetry, we need to find out the asymptotic behavior of $u_{i}$, which is proved in the next theorem. Notice that the $f_{i}$ are homogeneous and the integrability of the $u_{i}$ is equivalent to the integrability of $\bar{u}(x)=u_{1}+\cdots+u_{m}$. In fact, $\left|f_{i}(u)\right| \leq C \cdot|\bar{u}|^{p}$. Then there exists a function $R(x)$ satisfying

$$
0<R(x) \leq C,
$$

such that

$$
\bar{u}(x)=R(x) W_{\beta, \gamma}\left(\bar{u}^{p}\right) .
$$

Clearly, $\bar{u} \in L^{r}\left(R^{n}\right)$ is equivalent to $u_{i} \in L^{r}\left(R^{n}\right)$. The regularity results for (3) are derived in [26]. Letting $p=q$ in Theorem 2.1 of [26, we can also see that

$$
\bar{u} \in L^{r}\left(R^{n}\right), \quad \text { for } \quad \frac{1}{r} \in\left(0, \frac{n-\gamma \beta}{n(\gamma-1)}\right) .
$$

Moreover, according to Theorem 3.1 in [26, we also have

$$
\bar{u} \in L^{\infty}\left(R^{n}\right) .
$$


Theorem 1.2. Assuming $u_{i} \in L^{p+\gamma-1}\left(R^{n}\right)$ solves (11), $i=1,2, \ldots, m$, if $f_{i}$ is monotone nondecreasing with respect to the variables $u_{1}, u_{2}, \ldots, u_{m}$, then there exist positive constants $c$ and $C$, such that for sufficiently large $|x|$,

$$
c|x|^{-\frac{n-\beta \gamma}{\gamma-1}} \leq \bar{u}(x) \leq C|x|^{-\frac{n-\beta \gamma}{\gamma-1}} .
$$

Remark. For (2), the decay rate as in Theorem 1.2 holds naturally by the Kelvin transform. However, for (1), the Kelvin transform is not valid. In addition, the proof of Theorem 1.2 is different from the argument in [20, which estimates the decay rates there by using the radial symmetry of positive solutions. Now, we will apply the conclusion of Theorem 1.2 to establish the radial symmetry. We have to find another method to obtain the decay rate.

\section{Asymptotic estimate}

In this section, we prove Theorem 1.2.

Theorem 2.1. Let $u \in L^{p+\gamma-1}\left(R^{n}\right)$ and $u$ be a positive solution of (11). Then there exists a positive constant $c$, such that for large $|x|$,

$$
u_{i}(x) \geq \frac{c}{|x|^{\frac{n-\beta \gamma}{\gamma-1}}}, \quad i=1,2, \ldots, m .
$$

Proof. Clearly, $\int_{B_{1}(0)} f_{i}(u(y)) d y \geq c>0, i=1,2, \ldots, m$. Therefore, it follows that

$$
\begin{aligned}
u_{i}(x) & =\int_{0}^{\infty}\left[\frac{\int_{B_{t}(x)} f_{i}(u(y)) d y}{t^{n-\beta \gamma}}\right]^{\frac{1}{\gamma-1}} \frac{d t}{t} \\
& \geq \int_{|x|+1}^{\infty}\left[\frac{\int_{B_{1}(0)} f_{i}(u(y)) d y}{t^{n-\beta \gamma}}\right]^{\frac{1}{\gamma-1}} \frac{d t}{t} \\
& \geq c \int_{|x|+1}^{\infty} t^{-\frac{n-\beta \gamma}{\gamma-1}} \frac{d t}{t} \\
& \geq c|x|^{-\frac{n-\beta \gamma}{\gamma-1}} .
\end{aligned}
$$

Theorem 2.1 is proved.

Proposition 2.2. We claim that for $i=1,2, \ldots, m$,

$$
\lim _{|x| \rightarrow \infty} u_{i}(x)=0 \text {. }
$$

Proof. Take $x_{0} \in R^{n}$. By (9), $\|\bar{u}\|_{\infty}<\infty$. Thus, $\forall \varepsilon>0$, there exists $\delta \in(0,1)$ such that

$$
R(x) \int_{0}^{\delta}\left[\frac{\int_{B_{t}\left(x_{0}\right)} \bar{u}^{p}(z) d z}{t^{n-\beta \gamma}}\right]^{\frac{1}{\gamma-1}} \frac{d t}{t} \leq C\|\bar{u}\|_{\infty}^{\frac{p}{\gamma-1}} \int_{0}^{\delta} t^{\frac{\beta \gamma}{\gamma-1}} \frac{d t}{t}<\varepsilon .
$$

As $\left|x-x_{0}\right|<\delta$,

$$
\begin{aligned}
& \int_{\delta}^{\infty}\left[\frac{\int_{B_{t}\left(x_{0}\right)} f_{i}(u(z)) d z}{t^{n-\beta \gamma}}\right]^{\frac{1}{\gamma-1}} \frac{d t}{t} \\
& \leq \int_{\delta}^{\infty}\left[\frac{\int_{B_{t+\delta}(x)} f_{i}(u(z)) d z}{(t+\delta)^{n-\beta \gamma}}\right]^{\frac{1}{\gamma-1}}\left(\frac{t+\delta}{t}\right)^{\frac{n-\beta \gamma}{\gamma-1}+1} \frac{d(t+\delta)}{t+\delta} \\
& \leq C \int_{0}^{\infty}\left[\frac{\int_{B_{t}(x)} f_{i}(u(z)) d z}{t^{n-\beta \gamma}}\right]^{\frac{1}{\gamma-1}} \frac{d t}{t} \\
& \leq C u_{i}(x), \quad i=1,2, \ldots, m .
\end{aligned}
$$


Combining these estimates, we get

$$
\bar{u}\left(x_{0}\right)<\varepsilon+C \bar{u}(x), \quad \text { for } \quad\left|x-x_{0}\right|<\delta .
$$

Noting $\lim _{\left|x_{0}\right| \rightarrow \infty} \int_{B_{\delta}\left(x_{0}\right)} \bar{u}^{p+\gamma-1}(x) d x=0$ since $\bar{u} \in L^{p+\gamma-1}\left(R^{n}\right)$, we have

$$
\begin{aligned}
\bar{u}^{p+\gamma-1}\left(x_{0}\right) & =\left|B_{\delta}\left(x_{0}\right)\right|^{-1} \int_{B_{\delta}\left(x_{0}\right)} \bar{u}^{p+\gamma-1}\left(x_{0}\right) d x \\
& \leq C \varepsilon^{p+\gamma-1}+C\left|B_{\delta}\left(x_{0}\right)\right|^{-1} \int_{B_{\delta}\left(x_{0}\right)} \bar{u}^{p+\gamma-1}(x) d x \rightarrow 0
\end{aligned}
$$

when $\left|x_{0}\right| \rightarrow \infty$. This result means that (11) holds.

Theorem 2.3. Let $u_{i} \in L^{p+\gamma-1}\left(R^{n}\right)$ and $u$ be a positive solution of (1). Assume $\gamma \in\left(\frac{3 n}{2 n+\beta}, 2\right]$. Then there exists a positive constant $C$, such that for large $|x|$,

$$
\bar{u}(x) \leq \frac{C}{|x|^{\frac{n-\beta \gamma}{\gamma-1}}} .
$$

Proof.

Step 1. $\forall \rho>0$, take a cutoff function $\psi_{\rho}(x)$ as follows: $\psi(x) \in C_{0}^{\infty}\left(B_{2} \backslash B_{1}\right)$ satisfying

$$
\begin{array}{ll}
0 \leq \psi(x) \leq 1, & \text { for } \quad 1 \leq|x| \leq 2 \\
\psi(x)=1, & \text { for } \quad \frac{5}{4} \leq|x| \leq \frac{7}{4}
\end{array}
$$

Define $\psi_{\rho}(x)=\psi\left(\frac{x}{\rho}\right)$ and set $h(x)=\bar{u}(x)|x|^{n / p} \psi_{\rho}(x)$. Then either we can find $C>0$ such that for any $x$,

$$
h(x) \leq C
$$

where $C$ is independent of $\rho$, or there exists an increasing sequence $\left\{\rho_{j}\right\}_{j=1}^{\infty}$ satisfying $\lim _{j \rightarrow \infty} \rho_{j}=\infty$, with some $x_{j} \in B_{2 \rho_{j}} \backslash B_{\rho_{j}}$,

$$
\lim _{j \rightarrow \infty} h\left(x_{j}\right)=\infty \text {. }
$$

Step 2. If (14) is true, then for large $|x|$,

$$
\bar{u}(x) \leq C|x|^{-n / p} .
$$

Then by (6) and (16), we have

$$
R(x) \int_{0}^{\frac{|x|}{2}}\left(\frac{\int_{B_{t}(x)} \bar{u}^{p}(y) d y}{t^{n-\beta \gamma}}\right)^{\frac{1}{\gamma-1}} \frac{d t}{t} \leq \frac{C}{|x|^{\frac{n}{\gamma-1}}} \int_{0}^{\frac{|x|}{2}} t^{\frac{\beta \gamma}{\gamma-1}} \frac{d t}{t} \leq \frac{C}{|x|^{\frac{n-\beta \gamma}{\gamma-1}}} .
$$

On the other hand, (8) shows that $u \in L^{p}\left(R^{n}\right)$. Write $C_{p}=\|u\|_{p}^{p}$. Then

$$
R(x) \int_{|x| / 2}^{\infty}\left(\frac{\int_{B_{t}(x)} \bar{u}^{p}(y) d y}{t^{n-\beta \gamma}}\right)^{\frac{1}{\gamma-1}} \frac{d t}{t} \leq C C_{p}^{\frac{1}{\gamma-1}} \int_{|x| / 2}^{\infty} t^{-\frac{n-\beta \gamma}{\gamma-1}} \frac{d t}{t} \leq C|x|^{-\frac{n-\beta \gamma}{\gamma-1}} .
$$

Combining this result with (17), and using (7), we obtain

$$
\bar{u}(x)=R(x) W_{1, \gamma}\left(\bar{u}^{p}\right)(x) \leq C|x|^{-\frac{n-\beta \gamma}{\gamma-1}} .
$$

This is (13). 
Step 3. Let $x_{\rho}$ be the maximum point of $h(x)$ in $B_{2 \rho} \backslash B_{\rho}$. We will prove (13) under the assumption of (15). First, by (15) we can find $\delta>0$ which is independent of $\rho$, such that (cf. [19])

$$
\psi_{\rho}\left(x_{\rho}\right)>\delta
$$

Since $\psi$ is smooth, (18) shows that there exists a suitable small constant $\sigma>0$, such that $\psi_{\rho}(y)>\delta / 2$ for $\left|y-x_{\rho}\right|<\sigma\left|x_{\rho}\right|$. Hence, from $h(y) \leq h\left(x_{\rho}\right)$, we deduce that

$$
\bar{u}(y) \leq C \frac{\bar{u}\left(x_{\rho}\right)}{\psi_{\rho}(y)} \leq C(\delta) \bar{u}\left(x_{\rho}\right), \quad \text { as } \quad\left|y-x_{\rho}\right|<\sigma\left|x_{\rho}\right| .
$$

Equations (7) and (6) imply that

$$
\begin{aligned}
\bar{u}\left(x_{\rho}\right) \leq & C\left[\int_{0}^{\sigma\left|x_{\rho}\right|}\left(\frac{\int_{B_{t}\left(x_{\rho}\right)} \bar{u}^{p}(y) d y}{t^{n-\beta \gamma}}\right)^{\frac{1}{\gamma-1}} \frac{d t}{t}\right. \\
& \left.+\int_{\sigma\left|x_{\rho}\right|}^{\infty}\left(\frac{\int_{B_{t}\left(x_{\rho}\right)} \bar{u}^{p}(y) d y}{t^{n-\beta \gamma}}\right)^{\frac{1}{\gamma-1}} \frac{d t}{t}\right]:=C\left(I_{1}+I_{2}\right) .
\end{aligned}
$$

Clearly,

$$
I_{2} \leq C_{p}^{\frac{1}{\gamma-1}} \int_{\sigma\left|x_{\rho}\right|}^{\infty} t^{-\frac{n-\beta \gamma}{\gamma-1}} \frac{d t}{t} \leq C\left|x_{\rho}\right|^{-\frac{n-\beta \gamma}{\gamma-1}}
$$

Using (19), we obtain that, for $r \in\left(0, \sigma\left|x_{\rho}\right|\right)$,

$$
\begin{aligned}
I_{1} \leq & C \bar{u}\left(x_{\rho}\right)\left[\int_{0}^{r}\left(\frac{\int_{B_{t}\left(x_{\rho}\right)} \bar{u}^{p-\gamma+1}(y) d y}{t^{n-\beta \gamma}}\right)^{\frac{1}{\gamma-1}} \frac{d t}{t}\right. \\
& \left.+\int_{r}^{\sigma\left|x_{\rho}\right|}\left(\frac{\int_{B_{t}\left(x_{\rho}\right)} \bar{u}^{p-\gamma+1}(y) d y}{t^{n-\beta \gamma}}\right)^{\frac{1}{\gamma-1}} \frac{d t}{t}\right]:=C \bar{u}\left(x_{\rho}\right)\left(J_{1}+J_{2}\right) .
\end{aligned}
$$

By (11), for any $\varepsilon \in(0,1)$, we have

$$
J_{1} \leq \varepsilon^{\frac{p-\gamma+1}{\gamma-1}} \int_{0}^{r} t^{\frac{\beta \gamma}{\gamma-1}} \frac{d t}{t} \leq \frac{1}{4 C^{2}}
$$

as long as $\rho$ is sufficiently large. On the other hand, by Hölder's inequality and (9),

$$
\int_{B_{t}\left(x_{\rho}\right)} \bar{u}^{p-\gamma+1}(y) d y \leq\|\bar{u}\|_{s}^{p-\gamma+1}\left|B_{t}\left(x_{\rho}\right)\right|^{1-\frac{p-\gamma+1}{s}} \leq C t^{n-n(p-\gamma+1) / s},
$$

and hence

$$
J_{2} \leq C \int_{r}^{\sigma\left|x_{\rho}\right|} t^{\frac{\beta \gamma-n(p-\gamma+1) / s}{\gamma-1}} \frac{d t}{t} \leq \frac{1}{4 C^{2}}
$$

if $\rho$ is sufficiently large and $r$ is chosen suitably large. Substituting the estimates of $J_{1}$ and $J_{2}$ into (22), we obtain

$$
I_{1} \leq \frac{1}{2 C} \bar{u}\left(x_{\rho}\right)
$$

when $\rho$ is sufficiently large. Inserting this result and (21) into (20) yields

$$
\bar{u}\left(x_{\rho}\right) \leq C\left|x_{\rho}\right|^{-\frac{n-\beta \gamma}{\gamma-1}} .
$$

By (19), we obtain that as $\left|x-x_{\rho}\right|<\sigma\left|x_{\rho}\right|$,

$$
\bar{u}(x) \leq C \bar{u}\left(x_{\rho}\right) \leq C\left|x_{\rho}\right|^{-\frac{n-\beta \gamma}{\gamma-1}} \leq C|x|^{-\frac{n-\beta \gamma}{\gamma-1}} .
$$


Since $\rho$ is arbitrary, the result above still holds for all $x$ as long as $|x|$ is large. Then (13) is verified, and the proof of Theorem 2.3 is complete.

\section{RADIAL SYMMETRY}

In this section, we prove Theorem 1.1.

Choose a special direction, for example $x_{1}$. Write $x=\left(x_{1}, x^{\prime}\right)$ and set $H_{\lambda}=$ $\left\{x \in R^{n}: x_{1}<\lambda\right\}$. Let $x_{\lambda}=\left(2 \lambda-x_{1}, x^{\prime}\right)$ and $u^{\lambda}(x)=u\left(x^{\lambda}\right)$.

Step 1. Moving plane from negative infinity.

Set

$$
E_{t}:=\left\{t \mid \int_{B_{t}(x)} f_{i}\left(u^{\lambda}\right) d y \leq \int_{B_{t}(x)} f_{i}(u) d y\right\} .
$$

By the mean value theorem,

$$
u_{i}(x)-u_{i}^{\lambda}(x) \leq \frac{1}{\gamma-1} \int_{E_{t}} \frac{\int_{B_{t}(x)} f_{i}(u)-f_{i}\left(u^{\lambda}\right) d y}{t^{n-\gamma \beta}} \xi^{\frac{2-\gamma}{\gamma-1}} \frac{d t}{t} .
$$

For $x \in E_{t}$, we have

$$
\frac{\int_{B_{t}(x)} f_{i}\left(u^{\lambda}\right) d y}{t^{n-\gamma \beta}} \leq \xi \leq \frac{\int_{B_{t}(x)} f_{i}(u) d y}{t^{n-\gamma \beta}} .
$$

Thus, we have the following basic estimate:

$$
\begin{aligned}
& u_{i}(x)-u_{i}^{\lambda}(x) \\
& \leq \frac{1}{\gamma-1} \int_{E_{t}} \frac{\int_{B_{t}(x)} f_{i}(u)-f_{i}\left(u^{\lambda}\right) d y}{t^{n-\gamma \beta}}\left(\frac{\int_{B_{t}(x)} f_{i}(u) d y}{t^{n-\gamma \beta}}\right)^{\frac{2-\gamma}{\gamma-1}} \frac{d t}{t} \\
& \leq \frac{1}{\gamma-1} \int_{0}^{\infty} \frac{\int_{B_{t}(x) \cap D_{i}^{\lambda}} f_{i}(u)-f_{i}\left(u^{\lambda}\right) d y}{t^{n-\gamma \beta}}\left(\frac{\int_{B_{t}(x)} f_{i}(u) d y}{t^{n-\gamma \beta}}\right)^{\frac{2-\gamma}{\gamma-1}} \frac{d t}{t} .
\end{aligned}
$$

Define

$$
\begin{aligned}
& B_{i}^{\lambda}=\left\{x \in H_{\lambda}: u_{i}\left(x^{\lambda}\right)<u_{i}(x)\right\}, \\
& D_{i}^{\lambda}=\left\{x \in H_{\lambda}: f_{i}\left(u\left(x^{\lambda}\right)\right)<f_{i}(u(x))\right\} .
\end{aligned}
$$

We can restrict the integration region to $B_{t}(x) \backslash B_{t}\left(x^{\lambda}\right)$, which lies in $H_{\lambda}$, and then restrict to $\left(B_{t}(x) \backslash B_{t}\left(x^{\lambda}\right)\right) \cap D_{i}^{\lambda} \subseteq B_{t}(x) \cap D_{i}^{\lambda}$.

Now, investigate $f_{i}(u)-f_{i}\left(u^{\lambda}\right)$. Let

$$
w^{\lambda,+}(y)=\left(u(y)-u^{\lambda}(y)\right)^{+}, \quad \bar{u}(y)=u_{1}(y)+\cdots+u_{m}(y) .
$$

For any fixed $y \in D_{i}^{\lambda}$, there are two possibilities.

Case 1. $\frac{1}{2} u_{l}(y) \geq u_{l}^{\lambda}(y)$ for some $l$.

So $u_{l}(y) \leq 2\left(u_{l}(y)-u_{l}^{\lambda}(y)\right)$. Moreover, for $|y|$ large, $\bar{u}(y) \leq C u_{l}(y)$, since they have the same asymptotic behavior. Thus for large $|y|$ we have:

$$
\begin{aligned}
& f_{i}(u(y))-f_{i}\left(u^{\lambda}(y)\right) \leq f_{i}(u(y)) \leq C|\bar{u}(y)|^{p} \\
& \leq C|\bar{u}(y)|^{p-1}|\bar{u}(y)| \leq C|\bar{u}(y)|^{p-1} u_{l}(y) \leq C|\bar{u}(y)|^{p-1}\left|w^{\lambda,+}(y)\right| .
\end{aligned}
$$

Case 2. $u_{l}(y) \leq u_{l}^{\lambda}(y)$ for $l \in I . u_{l}(y) \geq u_{l}^{\lambda}(y) \geq \frac{1}{2} u_{l}(y)$ for $l \in J$. Here both $I$ and $J$ are subsets of $\{1, \ldots, m\}$. 
Since $y \in D_{i}^{\lambda}$ means $J$ is nonempty, without loss of generality, we assume $J=$ $\{1, \ldots, k\}$ for fixed $y$. Thus,

$$
\begin{aligned}
f_{i}(u(y)) & -f_{i}\left(u^{\lambda}(y)\right) \\
\leq & {\left[f_{i}\left(u_{1}, \ldots, u_{m}\right)-f_{i}\left(u_{1}^{\lambda}, u_{2}, \ldots, u_{m}\right)\right] } \\
& +\cdots+\left[f_{i}\left(u_{1}^{\lambda}, \ldots, u_{k-1}^{\lambda}, u_{k}, \ldots, u_{m}\right)-f_{i}\left(u_{1}^{\lambda}, \ldots, u_{k}^{\lambda}, u_{k+1}, \ldots, u_{m}\right)\right] \\
& +\left[f_{i}\left(u_{1}^{\lambda}, \ldots, u_{k}^{\lambda}, u_{k+1}, \ldots, u_{m}\right)-f_{i}\left(u_{1}^{\lambda}, \ldots, u_{m}^{\lambda}\right)\right] \\
\leq & {\left[f_{i}\left(u_{1}, \ldots, u_{m}\right)-f_{i}\left(u_{1}^{\lambda}, u_{2}, \ldots, u_{m}\right)\right] } \\
& +\cdots+\left[f_{i}\left(u_{1}^{\lambda}, \ldots, u_{k-1}^{\lambda}, u_{k}, \ldots, u_{m}\right)-f_{i}\left(u_{1}^{\lambda}, \ldots, u_{k}^{\lambda}, u_{k+1}, \ldots, u_{m}\right)\right] \\
\leq & \frac{\partial f_{i}\left(\xi_{1}, u_{2}, \ldots, u_{m}\right)}{\partial u_{1}} w_{1}^{\lambda,+}(y) \\
& +\cdots+\frac{\partial f_{i}\left(u_{1}^{\lambda}, \ldots, u_{k-1}^{\lambda}, \xi_{k}, u_{k+1}, \ldots, u_{m}\right)}{\partial u_{k}} w_{k}^{\lambda,+}(y) .
\end{aligned}
$$

Noticing that for $l \in J, u_{l}(y) \geq \xi_{l}(y) \geq \frac{1}{2} u_{l}(y)$, we can see that

$$
\frac{\partial f_{i}}{\partial u_{l}}\left(u_{1}^{\lambda}, \ldots, \xi_{l}, \ldots, u_{m}\right) \leq C \frac{f_{i}\left(u_{1}^{\lambda}, \ldots, \xi_{l}, \ldots, u_{m}\right)}{\xi_{l}} .
$$

Therefore,

$$
f_{i}(u(y))-f_{i}\left(u^{\lambda}(y)\right) \leq C|\bar{u}(y)|^{p-1}\left|w^{\lambda,+}(y)\right| .
$$

Combining these results into the previous estimate, we have

$$
\begin{aligned}
u_{i}(x) & -u_{i}^{\lambda}(x) \\
& \leq \frac{1}{\gamma-1} \int_{0}^{\infty} \frac{\int_{D_{i}^{\lambda} \cap B_{t}(x)} f_{i}(u)-f_{i}\left(u^{\lambda}\right) d y}{t^{n-\gamma \beta}}\left(\frac{\int_{B_{t}(x)} f_{i}(u) d y}{t^{n-\gamma \beta}}\right)^{\frac{2-\gamma}{\gamma-1}} \frac{d t}{t} \\
& \leq C \int_{0}^{\infty} \frac{\int_{D_{i}^{\lambda} \cap B_{t}(x)} \bar{u}(y)^{p-1} w^{\lambda,+}(y) d y}{t^{n-\gamma \beta}}\left(\frac{\int_{B_{t}(x)} f_{i}(u) d y}{t^{n-\gamma \beta}}\right)^{\frac{2-\gamma}{\gamma-1}} \frac{d t}{t} \\
& =C \int_{0}^{\infty} \frac{\int_{B_{t}(x)} \chi_{D_{i}^{\lambda}}(y) \bar{u}(y)^{p-1} w^{\lambda,+}(y) d y}{t^{n-\gamma \beta}}\left(\frac{\int_{B_{t}(x)} f_{i}(u) d y}{t^{n-\gamma \beta}}\right)^{\frac{2-\gamma}{\gamma-1}} \frac{d t}{t} \\
& \leq C\left(u_{i}\right)^{2-\gamma}\left(\int_{0}^{\infty}\left(\frac{\int_{B_{t}(x)} \chi_{D_{i}^{\lambda}}(y) \bar{u}(y)^{p-1} w^{\lambda,+}(y) d y}{t^{n-\gamma \beta}}\right)^{\frac{1}{\gamma-1}} \frac{d t}{t}\right)^{\gamma-1} \\
& :=C\left(u_{i}\right)^{2-\gamma} \cdot A^{\gamma-1} .
\end{aligned}
$$

By the Wolff inequality and the Hardy-Littlewood-Sobolev inequality, we have

$$
\begin{aligned}
\|A\|_{r}^{\gamma-1} & \leq C\left\|I_{\gamma \beta}\left(\bar{u}^{p-1} w^{\lambda,+} \chi_{D_{i}^{\lambda}}\right)\right\|_{\frac{r}{\gamma-1}} \\
& \leq C\left\|\bar{u}^{p-1} w^{\lambda,+} \chi_{D_{i}^{\lambda}}\right\|_{\frac{n r}{n(\gamma-1)+\gamma \beta r}} \\
& \leq\|\bar{u}\|_{p+\gamma-1, D_{i}^{\lambda}}^{p-1}\left\|w^{\lambda,+}\right\|_{s, D_{i}^{\lambda}},
\end{aligned}
$$

where $\frac{\gamma-1}{r}-\frac{1}{s}=\frac{p-1}{p+\gamma-1}-\frac{\gamma \beta}{n}$. We choose $r=p+\gamma-1$. Then all the requirements of the above inequalities are met, and moreover $s=p+\gamma-1$. Thus,

$$
\begin{aligned}
\left\|u_{i}-u_{i}^{\lambda}\right\|_{p+\gamma-1, D_{i}^{\lambda}} & \leq C\left\|u_{i}\right\|_{p+\gamma-1}^{2-\gamma}\|A\|_{p+\gamma-1}^{\gamma-1} \\
& \leq C\left\|u_{i}\right\|_{p+\gamma-1}^{2-\gamma}\|\bar{u}\|_{p+\gamma-1, D_{i}^{\lambda}}^{p-1}\left\|w^{\lambda,+}\right\|_{p+\gamma-1, D_{i}^{\lambda}} .
\end{aligned}
$$


Let $B^{\lambda}=\bigcup B_{i}^{\lambda}$ and $D^{\lambda}=\bigcup D_{i}^{\lambda}$. Then $D^{\lambda} \subset B^{\lambda}$. Therefore, we obtain the basic estimate

$$
\left\|w^{\lambda,+}\right\|_{p+\gamma-1, H_{\lambda}} \leq C\|\bar{u}\|_{p+\gamma-1}^{2-\gamma}\|\bar{u}\|_{p+\gamma-1, B^{\lambda}}^{p-1}\left\|w^{\lambda,+}\right\|_{p+\gamma-1, H_{\lambda}} .
$$

Now for $\lambda$ near negative infinity, $\|\bar{u}\|_{p+\gamma-1, B^{\lambda}}$ is so small that

$$
\|\bar{u}\|_{p+\gamma-1}^{2-\gamma}\|\bar{u}\|_{p+\gamma-1, B^{\lambda}}^{p-1} \leq \frac{1}{2 C} .
$$

Inserting this result into (23) yields

$$
\left\|w^{\lambda,+}\right\|_{p+\gamma-1, H_{\lambda}}=0 .
$$

In other words, $u_{i}(y) \leq u_{i}^{\lambda}(y)$ and $f_{i}(u(y)) \leq f_{i}\left(u^{\lambda}(y)\right)$ for almost all $y \in H_{\lambda}$.

Step 2. First, we introduce a simple lemma.

Lemma 3.1. Let $\Omega_{t}(x)=B_{t}(x) \backslash B_{t}\left(x^{\lambda}\right) \subset H_{\lambda}$. Then we have

$$
\begin{aligned}
u_{i}(x)-u_{i}^{\lambda}(x)= & \int_{0}^{\infty}\left(\frac{\int_{\Omega_{t}(x)} f_{i}(u(y)) d y}{t^{n-\gamma \beta}}\right)^{\frac{1}{\gamma-1}} \frac{d t}{t} \\
& -\int_{0}^{\infty}\left(\frac{\int_{\Omega_{t}(x)} f_{i}\left(u^{\lambda}(y)\right) d y}{t^{n-\gamma \beta}}\right)^{\frac{1}{\gamma-1}} \frac{d t}{t} .
\end{aligned}
$$

Define $\lambda_{0}=\sup \left\{\lambda \in R \mid \mu\left(B^{s}\right)=0\right.$, for all $\left.s \leq \lambda\right\}$.

Claim. If $u_{i}$ is not identically equal to $u_{i}^{\lambda_{0}}$, then $u_{i}(x)<u_{i}^{\lambda_{0}}(x), x \in H_{\lambda_{0}}$.

By Lemma 3.1, without loss of generality, we can assume there is some $k$ such that $f_{i}(u(x))<f_{i}\left(u^{\lambda_{0}}(x)\right)$ on a set of positive measure for $i \in\{1, \ldots, k\}$ and $f_{i}(u(x))=f_{i}\left(u^{\lambda_{0}}(x)\right)$ a.e. for $i \in\{k+1, \ldots, m\}$. Using Lemma 3.1 again, we have

$$
u_{i}(x)<u_{i}^{\lambda_{0}}(x), \quad \text { as } \quad x \in H_{\lambda_{0}}, \quad \text { for } \quad i \in\{1, \ldots, k\}
$$

and

$$
u_{i}(x)=u_{i}^{\lambda_{0}}(x) \text { a.e. for } i \in\{k+1, \ldots, m\} .
$$

If $k=m$, the claim is proved. If $k<m,\left\{u_{1}, \ldots, u_{m}\right\} \neq\left\{u_{1}^{\lambda_{0}}, \ldots, u_{m}^{\lambda_{0}}\right\}$ on a positive measure set and $u \leq u^{\lambda_{0}}$. Since the system is simple, we must have $\left\{f_{k+1}(u), \ldots, f_{m}(u)\right\} \neq\left\{f_{k+1}\left(u^{\lambda_{0}}\right), \ldots, f_{m}\left(u^{\lambda_{0}}\right)\right\}$ on a positive measure set; that is, $f_{i}(u(x)) \leq f_{i}\left(u^{\lambda_{0}}(x)\right)$ on a positive measure set. This contradicts our assumption.

By this claim, we can move the plane further to some $\lambda \geq \lambda_{0}$, which contradicts our assumption. First, we assume that the $L^{p+\gamma-1}$ norm of $\bar{u}$ outside some fixed bounded region $B$ is small. Since

$$
w^{\lambda}(x)=u(x)-u^{\lambda}(x) \rightarrow w^{\lambda_{0}}
$$

in the norm of $L^{p+\gamma-1}(B), w^{\lambda}(x)$ converges to $w^{\lambda_{0}}$ in measure (here we always restrict our attention in $B$ ). Let

$$
A_{\delta}=\left\{x \in B \cap H_{\lambda_{0}} \mid 0>w^{\lambda_{0}}(x)>-\delta\right\} .
$$

By virtue of $w^{\lambda_{0}}<0$ on $B \cap H_{\lambda_{0}}$, for any given $\varepsilon>0, \mu\left(A_{\delta}\right)<\varepsilon$ for some $\delta$ small enough. When $\lambda$ is close enough to $\lambda_{0}, B \cap\left(H_{\lambda}-H_{\lambda_{0}}\right)$ has small measure. Since $w^{\lambda}$ converges to $w^{\lambda^{0}}$ in measure, we have $w^{\lambda}(x)<0$ on $\left(B \cap H_{\lambda_{0}}-A_{\delta}\right)$ except for a small measure subset, say $E$. Thus, combining all the above estimates, we get

$$
\left\{x: w^{\lambda}(x)>0\right\} \subseteq B^{C} \cup\left(B \cap\left(H_{\lambda}-H_{\lambda_{0}}\right)\right) \cup E \cup A_{\delta} .
$$


Therefore, we can still deduce that $\|\bar{u}\|_{p+\gamma-1, B^{\lambda}}$ is sufficiently small. Theorem 1.2, together with (9), implies that for any $\delta>0$, there exist two positive constants $c, C$ such that

$$
c \leq u_{i}(y) \leq C, \quad \forall y \in R^{n} \backslash B_{\delta}(x) .
$$

There are two cases. If $\lambda_{0}<0$, then we can still apply the basic estimate (23) to derive $u(x) \leq u_{\lambda}(x)$ when $\lambda$ is close to $\lambda_{0}$. This leads to a contradiction, which implies that $u(x)=u^{\lambda_{0}}(x)$ almost everywhere. If $\lambda_{0}>0$, the moving plane can stop at $x_{1}=0$. Then we repeat the above argument from $x_{1}=\infty$. Now, we also have two cases. One is that the plane stops before the origin. The argument is the same as in the first case. The other case is that the plane stops at the origin, which still shows that $u(x)=u_{0}^{\lambda}(x)$. Finally, since the direction $x_{1}$ is chosen arbitrarily, we know that $u_{1}(x), u_{2}(x), \ldots, u_{m}(x)$ are radially symmetric and decreasing about the same point $x_{0}$. This completes our proof.

\section{ACKNOWLEDGMENTS}

The authors are grateful to Prof. Congming Li for his continued encouragement. The work of the first author was supported partly by NSF of China (No. 11171158).

\section{REFERENCES}

[1] L. Caffarelli, B. Gidas, and J. Spruck, Asymptotic symmetry and local behavior of semilinear elliptic equations with critical Sobolev growth, Comm. Pure Appl. Math., 42 (1989), 271-297. MR.982351 (90c:35075)

[2] W. Chen and C. Li, Classification of solutions of some nonlinear elliptic equations, Duke Math. J., 63 (1991), 615-622. MR.1121147 (93e:35009)

[3] W. Chen and C. Li, A priori estimates for prescribing scalar curvature equations, Ann. of Math. (2), 145 (1997), 547-564. MR1454703 (98d:53049)

[4] W. Chen and C. Li, The best constant in a weighted Hardy-Littlewood-Sobolev inequality, Proc. Amer. Math. Soc., 136 (2008), 955-962. MR2361869 (2009b:35098)

[5] W. Chen and C. Li, Radial symmetry of solutions for some integral systems of Wolff type, Disc. Cont. Dynamics Sys., 30 (2011), 1083-1093.

[6] W. Chen and C. Li, Classification of positive solutions for nonlinear differential and integral systems with critical exponents, Acta Mathematica Scientia, 29B (2009), 949-960. MR2510000 (2010i:35078)

[7] W. Chen, C. Li, and B. Ou, Classification of solutions for a system of integral equations, Comm. in Partial Differential Equations, 30 (2005), 59-65. MR.2131045 (2006a:45007)

[8] W. Chen, C. Li, and B. Ou, Classification of solutions for an integral equation, Comm. Pure and Appl. Math., 59 (2006), 330-343. MR2200258 (2006m:45007a)

[9] C. Cascante, J. Ortega, and I. Verbitsky, Wolff's inequality for radially nonincreasing kernels and applications to trace inequalities, Potential Analysis, 16 (2002), 347-372. MR 1894503 (2003f:31006a)

[10] S-Y. A. Chang and P. Yang, On uniqueness of an $n$-th order differential equation in conformal geometry, Math. Res. Letters, 4 (1997), 91-102. MR1432813 (97m:58204)

[11] L. Fraenkel, An Introduction to Maximum Principles and Symmetry in Elliptic Problems, Cambridge University Press, Cambridge, 2000. MR.1751289 (2001c:35042)

[12] B. Gidas, W. M. Ni, and L. Nirenberg, Symmetry of positive solutions of nonlinear elliptic equations in $R^{n}$, collected in Mathematical Analysis and Applications, vol. 7a of the book series Advances in Mathematics. Supplementary Studies, Academic Press, New York, 1981. MR634248 (84a:35083)

[13] L.I. Hedberg and T. Wolff, Thin sets in nonlinear potential theory, Ann. Inst. Fourier (Grenoble), 33 (1983), 161-187. MR727526 (85f:31015)

[14] C. Jin and C. Li, Symmetry of solutions to some systems of integral equations, Proc. Amer. Math. Soc., 134 (2006), 1661-1670. MR2204277 (2006j:45017)

[15] C. Jin and C. Li, Qualitative analysis of some systems of integral equations, Calc. Var. PDEs, 26 (2006), 447-457. MR2235882 (2007c:45013) 
[16] T. Kilpelaiinen and J. Maly, Degenerate elliptic equations with measure data and nonlinear potentials, Ann. Scuola Norm. Sup. Pisa, Cl. Sci., 19 (1992), 591-613. MR 1205885 (94c:35091)

[17] T. Kilpelaiinen and J. Maly, The Wiener test and potential estimates for quasilinear elliptic equations, Acta Math., 172 (1994), 137-161. MR.1264000 (95a:35050)

[18] D. Labutin, Potential estimates for a class of fully nonlinear elliptic equations, Duke Math. J., 111 (2002), 1-49. MR 1876440 (2002m:35053)

[19] Y. Lei, C. Li, and C. Ma, Decay estimation for positive solutions of a $\gamma$-Laplace equation, Discrete Contin. Dyn. Syst., 30 (2011), 547-558. MR2772129

[20] Y. Lei and C. Ma, Asymptotic behavior for solutions of some integral equations, Comm. Pure Appl. Anal. 10 (2011), 193-207. MR2746534

[21] C. Li, Local asymptotic symmetry of singular solutions to nonlinear elliptic equations, Invent. Math., 123 (1996), 221-231. MR1374197 (96m:35085)

[22] C. Li and J. Lim, The singularity analysis of solutions to some integral equations, Comm. Pure Appl. Anal., 6 (2007), 453-464. MR 2289831 (2008e:45008)

[23] C. Li and L. Ma, Uniqueness of positive bound states to Schrödinger systems with critical exponents, SIAM J. Math. Anal., 40 (2008), 1049-1057. MR2452879 (2009k:35079)

[24] Y. Li, Remark on some conformally invariant integral equations: the method of moving spheres, Journal of European Mathematical Society, 6 (2004), 153-180. MR2055032 (2005e:45007)

[25] E. Lieb, Sharp constants in the Hardy-Littlewood-Sobolev and related inequalities, Ann. of Math. (2) 118 (1983), 349-374. MR717827 (86i:42010)

[26] C. Ma, W. Chen, and C. Li, Regularity of solutions for an integral system of Wolff type, Adv. Math., 226 (2011), 2676-2699. MR2739789

[27] J. Maly, Wolff potential estimates of superminimizers of Orlicz type Dirichlet integrals, Manuscripta Math., 110 (2003), 513-525. MR1975101(2004i:35073)

[28] N. Phuc and I. Verbitsky, Quasilinear and Hessian equations of Lane-Emden type, Ann. of Math. (2) 168 (2008), 859-914. MR2456885 (2010a:35075)

[29] J. Serrin, A symmetry problem in potential theory, Arch. Rational Mech. Anal., 43 (1971), 304-318. MR0333220 (48:11545)

[30] J. Serrin and H. Zou, Cauchy-Liouville and universal boundedness theorems for quasilinear elliptic equations and inequalities, Acta Math., 189 (2002), 79-142. MR.1946918|(2003j:35107)

[31] E. M. Stein and G. Weiss, Fractional integrals in n-dimensional Euclidean space, J. Math. Mech., 7 (1958), 503-514. MR0098285 (20:4746)

Institute of Mathematics, School of Mathematical Sciences, Nanjing Normal UniVersity, Nanjing, 210097, People's Republic of China

E-mail address: leiyutian@njnu.edu.cn

Department of Mathematics, University of Colorado at Boulder, Boulder, ColoRADO 80309

E-mail address: chao.ma@colorado.edu 